\title{
Online Estimation of ARW Coefficient of Fiber Optic Gyro
}

\author{
Yang Li, Baiqing Hu, Fangjun Qin, and Kailong Li \\ Electrical Engineering College, Naval University of Engineering, Wuhan 430033, China \\ Correspondence should be addressed to Yang Li; hgdh_ly@126.com
}

Received 22 October 2013; Revised 13 April 2014; Accepted 24 April 2014; Published 20 May 2014

Academic Editor: Slim Choura

Copyright (C) 2014 Yang Li et al. This is an open access article distributed under the Creative Commons Attribution License, which permits unrestricted use, distribution, and reproduction in any medium, provided the original work is properly cited.

\begin{abstract}
As a standard method for noise analysis of fiber optic gyro (FOG), Allan variance has too large offline computational burden and data storages to be applied to online estimation. To overcome the barriers, the state space model is firstly established for FOG. Then the Sage-husa adaptive Kalman filter (SHAKF) is introduced in this field. Through recursive calculation of measurement noise covariance matrix, SHAKF can avoid the storage of large amounts of history data. However, the precision and stability of this method are still the primary matters that needed to be addressed. Based on this point, a new online method for estimation of the coefficient of angular random walk is proposed. In the method, estimator of measurement noise is constructed by the recursive form of Allan variance at the shortest sampling time. Then the estimator is embedded into the SHAKF framework resulting in a new adaptive filter. The estimations of measurement noise variance and Kalman filter are independent of each other in this method. Therefore, it can address the problem of filtering divergence and precision degrading effectively. Test results of both digital simulation and experimental data of FOG verify the validity and feasibility of the proposed method.
\end{abstract}

\section{Introduction}

Fiber optic gyro (FOG) random noise has vital effect on the performance of FOG in the field of navigation and aviation. As one of the most important components of FOG random noise, angular random walk (ARW) is generally used to quantitatively characterize the intensity of white noise of FOG output signal. Estimation of ARW coefficient of FOG is useful for the performance improvement of on-board attitude determination filter [1]. In some sense, a good knowledge of ARW of FOG becomes the key information to evaluate the state of FOG.

To determine the ARW coefficient of FOG, the Allan variance method is commonly adopted as a standard $[2,3]$. For example, Allan variance method is recommended by the United States IEEE (Std. 952-1997, R2008) and China's military standard (GJB 2426A-2004) to calculate the ARW coefficient. Allan variance was initially developed by David Allan of the National Bureau of Standards to quantify the error statistics for a Caesium beam frequency standard. The method, in general, can be applied to analyze the error characteristics of any precise measurement instruments. In nature, it is offline and requires a large amount of static data of
FOG to be stored [4]. Besides, it requires a procedure of data section selection to obtain accuracy estimations of the noise contributions, but the selection is usually done manually.

To calculate ARW coefficient online and decrease the requirement of data store, some methods have been proposed [5-10]. In [7, 8], coefficient of ARW is estimated for inflight gyros, but they are offline in nature. Additionally, other methods presented in $[9,10]$ estimate ARW coefficient by using nonlinear adaptive filter technique, but the state-space model is difficult to be established.

Focusing on the disadvantages and requirements, the Sage-husa adaptive Kalman filter (SHAKF) method [1114] is firstly introduced into this field, which can estimate statistical characteristic online through the measurement output while estimating state and is considered as the most promising method for general online applications $[15$, 16]. In particular, the SHAKF method is widely used in seam tracking monitoring, signal denoising, and integrated navigation system [17-19]. Through recursive calculation of measurement noise covariance matrix, this method can avoid the storage of large amounts of history data and therefore greatly reduce the computational burden for processing gyro data. However, the precision and stability of the SHAKF are 
still the primary matters that needed to be addressed [2022 ]. Based on this point, a new online method for estimation of the coefficient of angular random walk is presented. In the proposed method, estimator of measurement noise is constructed by the recursive form of Allan variance at the shortest sampling time. Then the estimator is embedded into the framework of SHAKF resulting in a new adaptive filter. The processes of measurement noise variance estimation and Kalman filter (KF) are independent of each other in this method. Therefore, it can address the problem of filtering divergence and precision degrading effectively.

The rest of the paper is organized as follows. Section 2 presents the state space model of the online estimation method and gives the implementation of the proposed method. Section 3 presents the experimental results. A digital simulation test and an experimental data test are done in this section to test the performance of the SHAKF method and the proposed method compared with the classical Allan variance method. Finally, conclusions are drawn in Section 4.

\section{Principle of Online Estimation}

2.1. Establishment of the State Space Model. According to Allan variance analysis, generally there are five error sources existing in FOG (IEEE Std. 952-1997, R2008) [2]: ARW, quantization noise, bias instability, angular rate random walk, and ramp noise. The noise parameters of these stochastic errors can be determined by Allan variance analysis, and these parameters can be used to determine the power spectral density (PSD) of the corresponding noises [10]. The Allan variances and PSDs of noises are listed in Table 1.

For bias instability, it will be significantly reduced after increasing the rate of data sampling. For ramp noise, it is essentially a deterministic error and is only present at low frequencies. Therefore, in the mathematical model, only ARW, bias instability, and angular rate random walk are considered for model simplifying.

Then, given the PSD of a stochastic process, the transfer function can be obtained by the following equation:

$$
S(j \omega)=|G(j \omega)|^{2}
$$

where $S(j \omega)$ is the PSD of a stochastic process, $G(j \omega)$ is the transfer function, and $\omega$ is the circular frequency and equals $2 \pi f$ with $f$ being the sampling rate. According to (1) and the PSD of $N, B$, and $K$, the transfer functions for $N, B$, and $K$ are given by

$$
\begin{gathered}
G_{a r \omega}(j \omega)=N \\
G_{b s}(j \omega)=\frac{B}{\sqrt{j \omega}} \\
G_{r r \omega}(j \omega)=\frac{K}{j \omega} .
\end{gathered}
$$

In low frequency band $[0.1 \mathrm{~Hz}, 10 \mathrm{~Hz}]$, the approximate transfer function of the second term of (2) is

\begin{tabular}{|c|c|c|c|}
\hline Noise types & Noise Coefficient & Allan variance $\left(\sigma^{2}(\tau)\right)$ & PSD \\
\hline $\begin{array}{l}\text { Angle } \\
\text { random walk }\end{array}$ & $N$ & $N^{2} / \tau$ & $N^{2}$ \\
\hline $\begin{array}{l}\text { Quantization } \\
\text { noise }\end{array}$ & Q & $3 Q^{2} / \tau^{2}$ & $(2 \pi f)^{2} Q^{2} T$ \\
\hline $\begin{array}{l}\text { Bias } \\
\text { instability }\end{array}$ & $B$ & $2 B^{2} \ln 2 / \pi$ & $\left(B^{2} / 2 \pi\right) / f$ \\
\hline $\begin{array}{l}\text { Angular rate } \\
\text { random walk }\end{array}$ & K & $K^{2} \tau / 3$ & $(K / 2 \pi)^{2} / f^{2}$ \\
\hline Ramp noise & $R$ & $R^{2} \tau^{2} / 2$ & $R^{2} /(2 \pi f)^{3}$ \\
\hline
\end{tabular}

$$
G_{b s}(j \omega)=\frac{\beta \cdot B}{j \omega+\beta} .
$$

TABLE 1: Random noise sources in FOG.

According to the theory of linear system, $N, B$, and $K$ can be described by the following three equations:

$$
\begin{gathered}
Y_{a r \omega}(j \omega)=N \cdot X_{a r \omega}(j \omega) \\
Y_{b s}(j \omega) \approx\left[\frac{(\beta \cdot B)}{(j \omega+\beta)}\right] \cdot X_{b s}(j \omega) \\
Y_{r r \omega}(j \omega)=\left(\frac{K}{j \omega}\right) \cdot X_{r r \omega}(j \omega),
\end{gathered}
$$

where $Y_{a r w}(j \omega), Y_{b s}(j \omega)$, and $Y_{r r w}(j \omega)$ are the Fourier transformation of system outputs and $X_{a r \omega}(j \omega), X_{b s}(j \omega)$, and $X_{r r \omega}(j \omega)$ are the Fourier transformation of system inputs. Then, the time-domain form of (4) is written as

$$
\begin{gathered}
y_{a r w}(t)=N \cdot u_{\text {arw }} \\
\dot{y}_{b s}(t)=-\beta \dot{y}_{b s}(t)+\beta \cdot B \cdot u_{b s} \\
\dot{y}_{r r w}(t)=K \cdot u_{r r \omega},
\end{gathered}
$$

where $u_{a r \omega}, u_{b s}$, and $u_{r r \omega}$ are the independent white noise. According to the differential equations, the corresponding discrete-time forms are

$$
\begin{gathered}
y_{a r w}(k)=\left(\frac{N}{\sqrt{\Delta T}}\right) \cdot u_{a r \omega} \\
y_{b s}(k)=(1-\beta \Delta T) y_{b s}(k-1)+\beta \cdot \sqrt{\Delta T} \cdot B \cdot u_{b s} \\
y_{r r w}(k)=y_{r r w}(k-1)+K \cdot \sqrt{\Delta T} \cdot u_{r r \omega} .
\end{gathered}
$$

Then filtering state equation and observation equation can be written as follows:

$$
\begin{aligned}
{\left[\begin{array}{c}
y_{r r w}(k) \\
y_{b s}(k)
\end{array}\right]=} & {\left[\begin{array}{cc}
1 & 0 \\
0 & (1-\beta \Delta T)
\end{array}\right] \cdot\left[\begin{array}{c}
y_{r r w}(k-1) \\
y_{b s}(k-1)
\end{array}\right] } \\
& +\left[\begin{array}{cc}
K \cdot \sqrt{\Delta T} & 0 \\
0 & \beta \cdot \sqrt{\Delta T} \cdot B
\end{array}\right] \cdot\left[\begin{array}{c}
u_{r r \omega} \\
u_{b s}
\end{array}\right] \\
z(k)= & {\left[\begin{array}{ll}
1 & 1
\end{array}\right] \cdot\left[\begin{array}{c}
y_{r r w}(k) \\
y_{b s}(k)
\end{array}\right]+\frac{N}{\sqrt{\Delta T}} \cdot u_{a r \omega} . }
\end{aligned}
$$



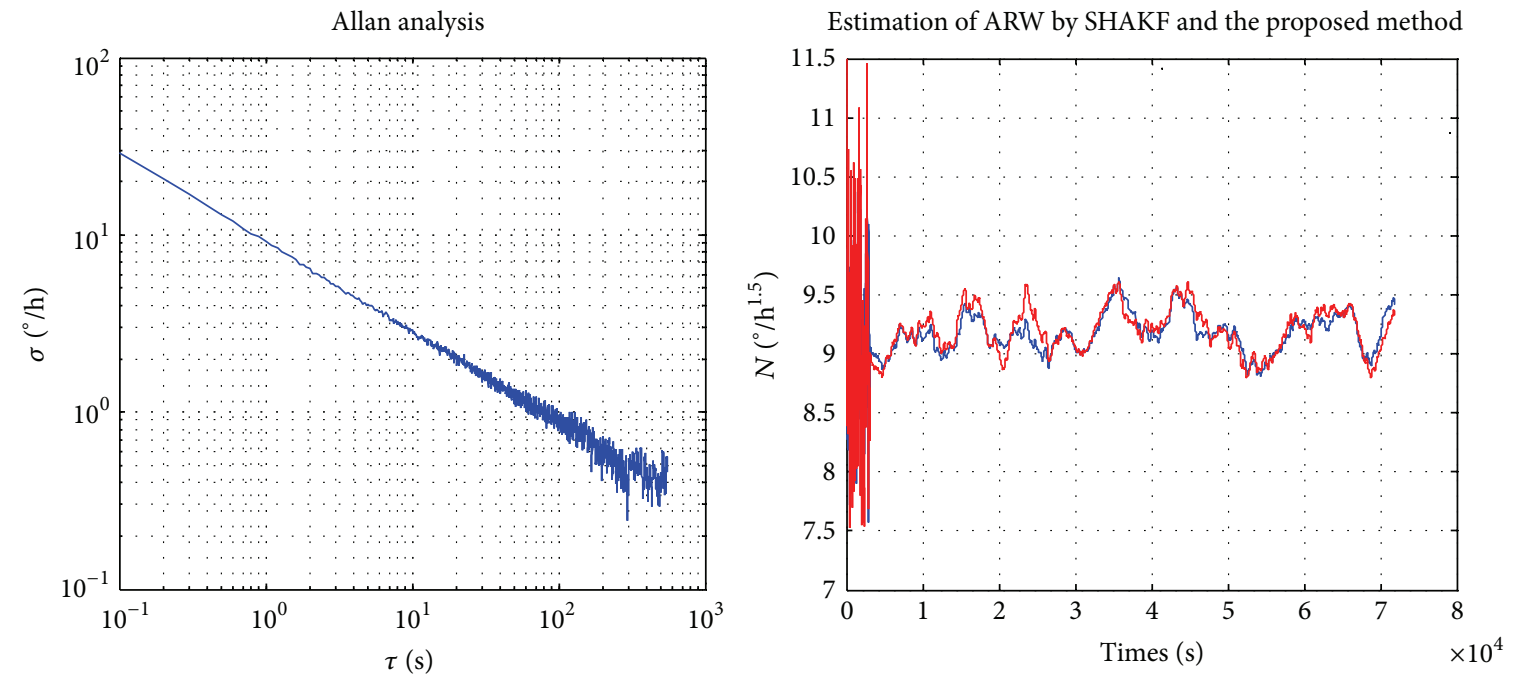

(a) $N=9.2000^{\circ} / \mathrm{h}^{1.5}$
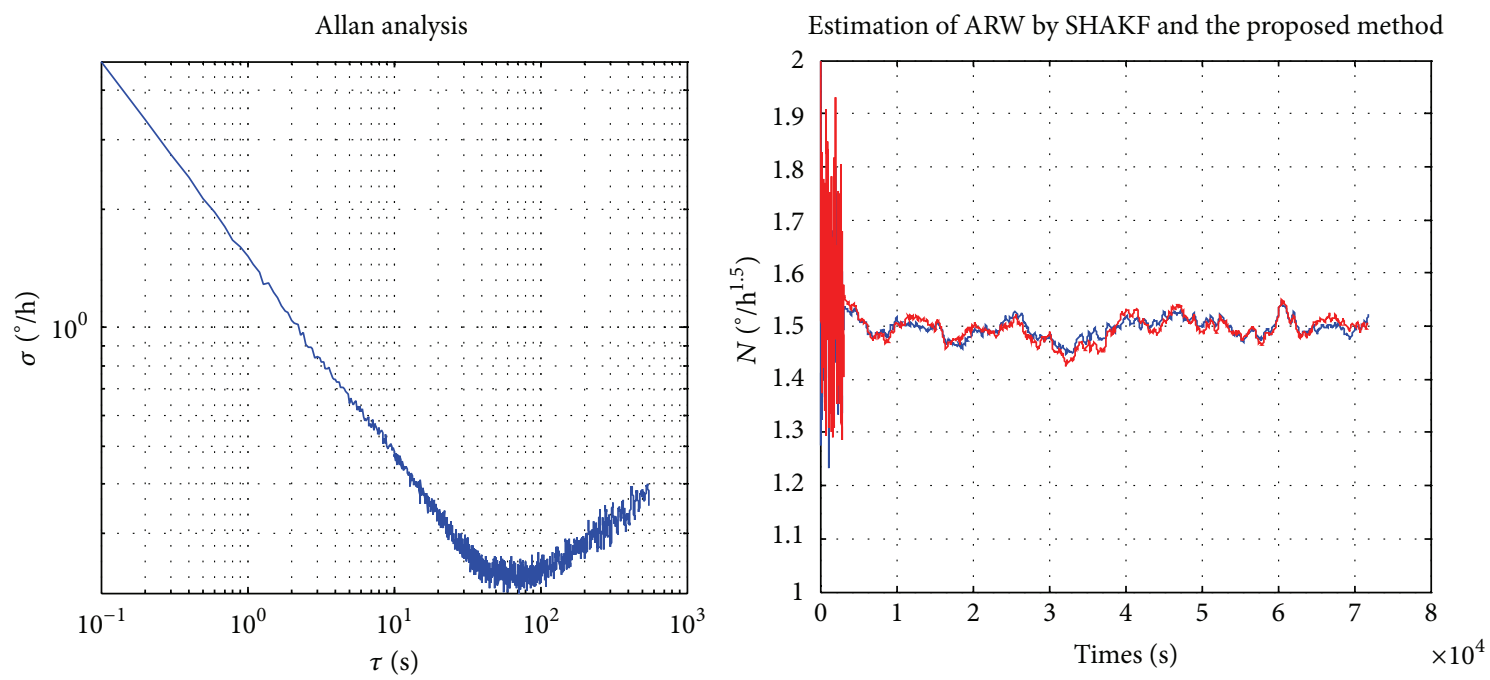

(b) $N=1.5000^{\circ} / \mathrm{h}^{1.5}$
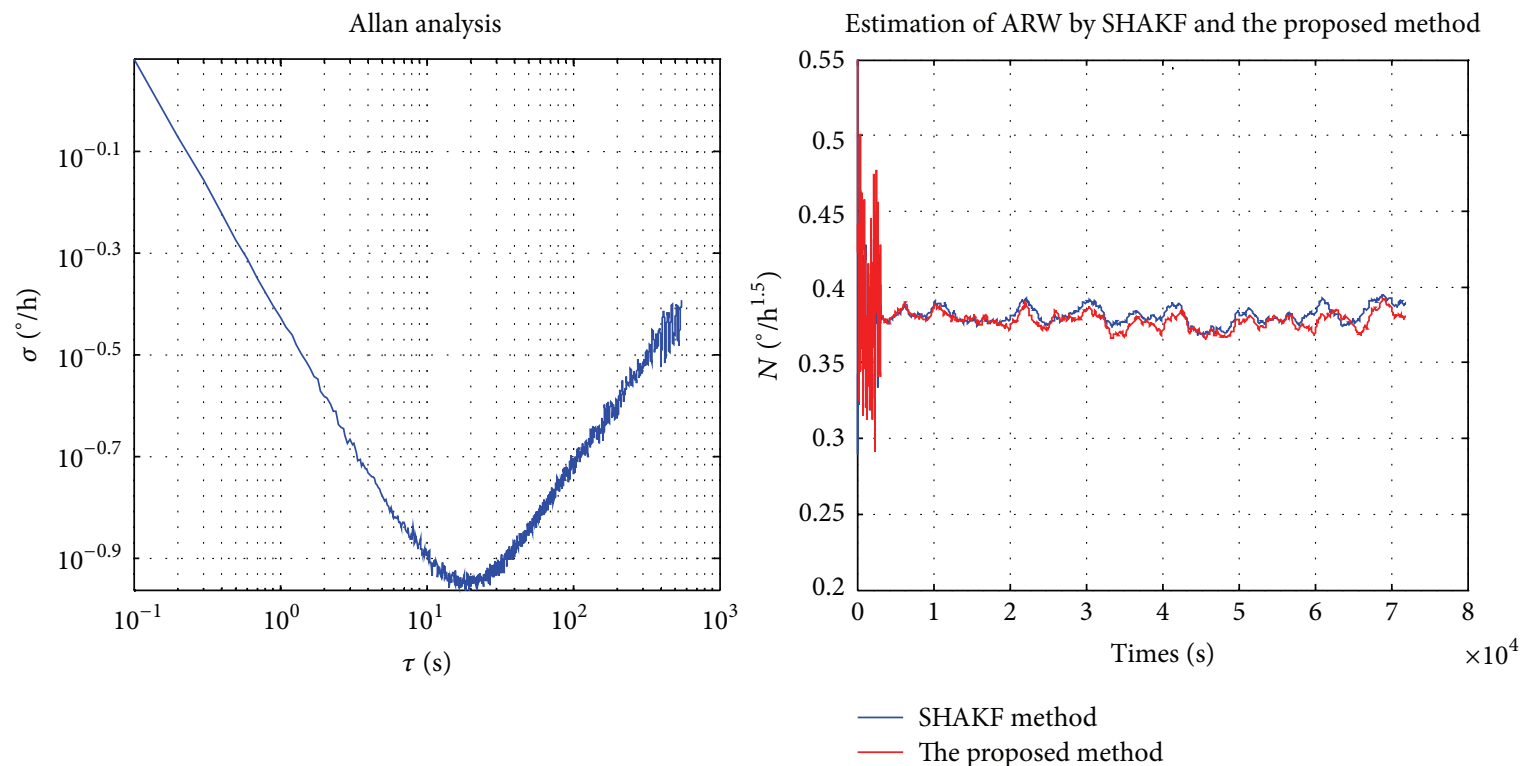

(c) $N=0.3800^{\circ} / \mathrm{h}^{1.5}$

FIgure 1: Continued. 

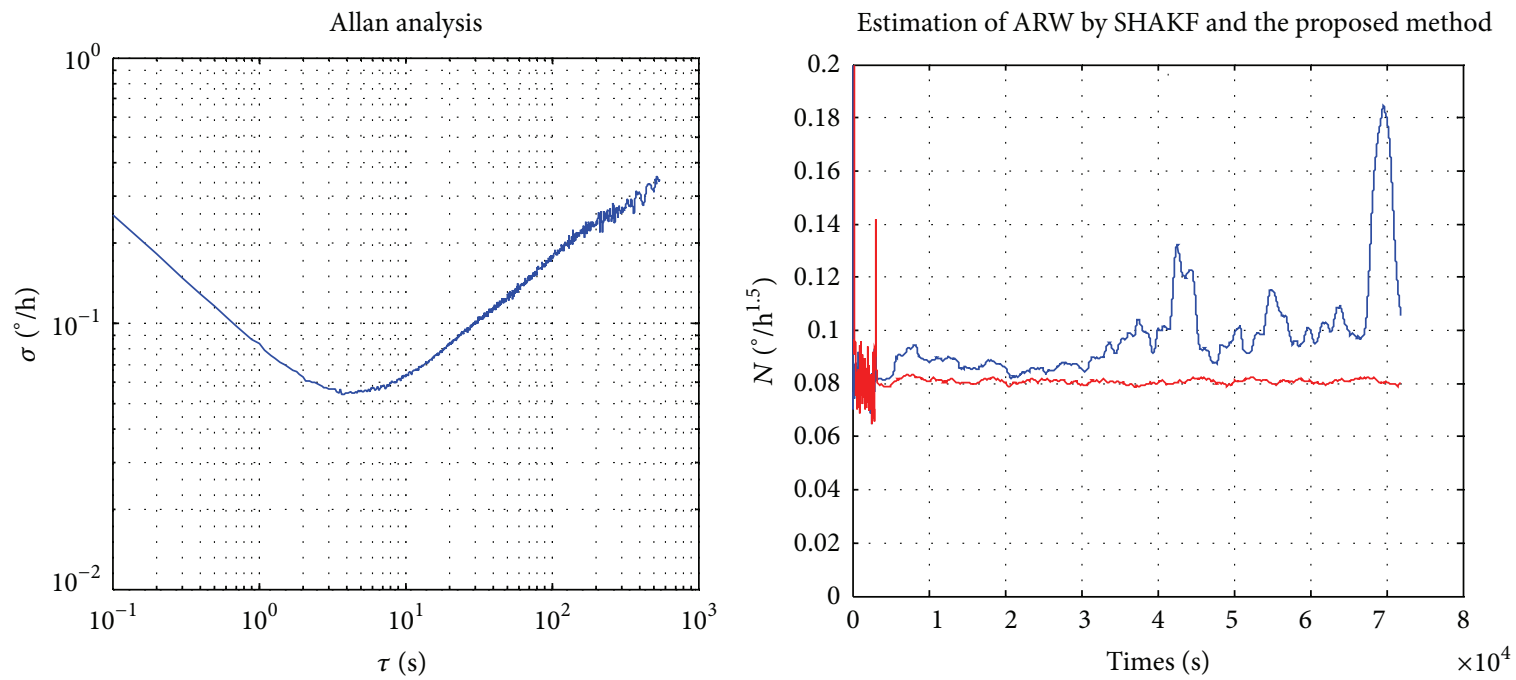

(d) $N=0.0810^{\circ} / \mathrm{h}^{1.5}$
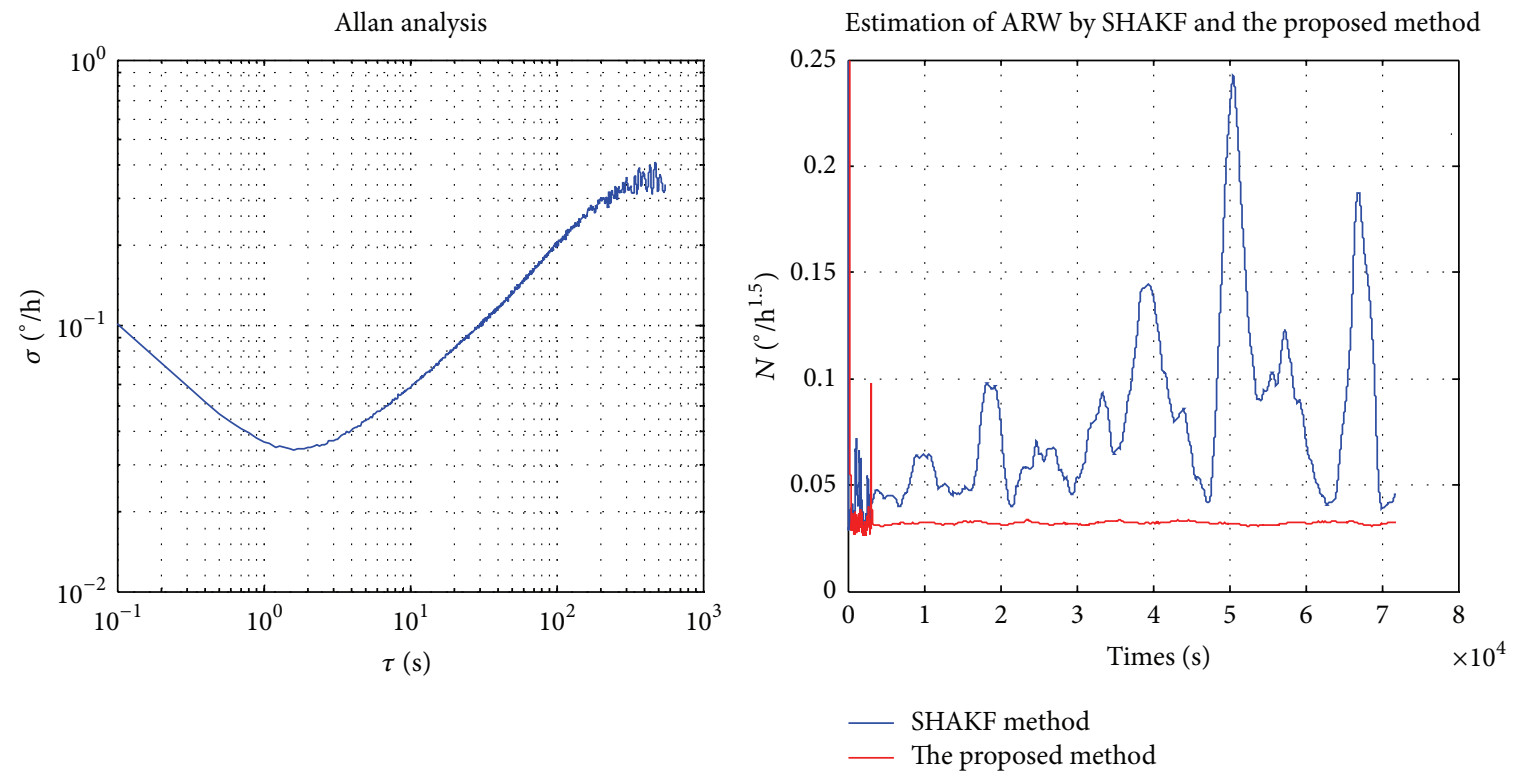

(e) $N=0.0320^{\circ} / \mathrm{h}^{1.5}$

FIGURE 1: Comparison of ARW estimation in different simulation data.

2.2. Online Estimation Methods. In order to facilitate the following discussion, (7a) and (7b) can be written in a compact form as

$$
\begin{gathered}
X_{k}=\Phi_{k-1} X_{k-1}+B_{k-1} w_{k-1} \\
Z_{k}=H_{k} X_{k}+v_{k},
\end{gathered}
$$

where $X_{k}=\left[\begin{array}{c}y_{r r w}(k) \\ y_{b s}(k)\end{array}\right]$ is state vector at time $k, w_{k-1}=\left[\begin{array}{c}u_{r r w} \\ u_{b s}\end{array}\right]$ is a Gauss white noise vector at time $k$, and its covariance matrix is $Q_{k}$. $Z_{k}$ is measurement vector at time $k, v_{k}=u_{\text {arw }}$ is measurement noise vector at time $k$, and its covariance matrix is $R_{k} . \Phi_{k-1}, B_{k-1}$, and $H_{k}$ can be easily obtained through (7a) and $(7 \mathrm{~b})$.
The process noise in (8) and measurement noise in (9) are assumed to be independent Gaussian noise with means and covariance matrices [19]

$$
\begin{gathered}
E\left[w_{k}\right]=q, \quad \operatorname{Cov}\left[w_{k}, w_{j}\right]=E\left[w_{j} w_{k}^{T}\right]=Q \delta_{j k} \\
E\left[v_{k}\right]=r, \quad \operatorname{Cov}\left[v_{k}, v_{j}\right]=E\left[v_{j} v_{k}^{T}\right]=R \delta_{j k} \\
\operatorname{Cov}\left[w_{j}, v_{k}\right]=E\left[w_{j} v_{k}^{T}\right]=0,
\end{gathered}
$$

where $E[\cdot]$ denotes the expected values, $\operatorname{Cov}[\cdot]$ denotes the covariance values, and $\delta_{j k}$ denotes the Kronecker delta function.

2.2.1. Sage-Husa Adaptive Kalman Filter. In the process of the SHAKF, the system states are estimated by innovations; at the 


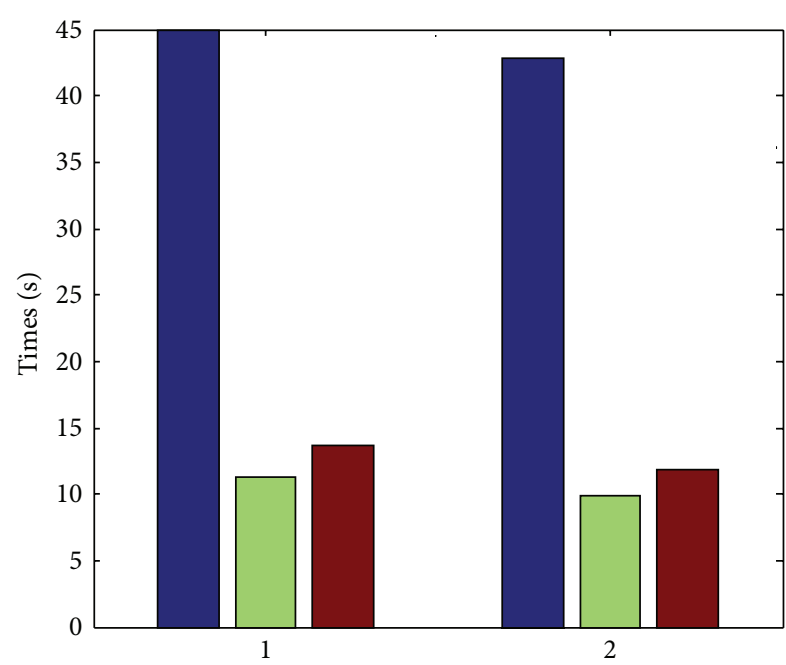

Allan method

SHAKF method

The proposed method

FIGURE 2: Comparison of total calculation time $\left(1: N=0.3800^{\circ} / \mathrm{h}^{1.5}\right.$, 2: $\left.N=9.2000^{\circ} / \mathrm{h}^{1.5}\right)$.

same time, the unknown noise statistics are modified. As the approximating values of $q, Q, r$, and $R$ are updated in realtime, the KF can get more accurate information of noise to estimate the states exactly.

For the state space models (8) and (9) with unknown statistic noise, the explicit procedure of SHAKF is given as follows:

$$
\begin{gathered}
\widehat{X}_{k, k-1}=\Phi_{k-1} \widehat{X}_{k-1}+B_{k-1} q_{k-1} \\
P_{k, k-1}=\Phi_{k-1} P_{k-1} \Phi_{k-1}^{T}+B_{k-1} Q_{k-1} B_{k-1}^{T} \\
K_{k}=P_{k, k-1} H_{k}^{T}\left(H_{k} P_{k, k-1} H_{k}^{T}+R_{k}\right)^{-1} \\
\widehat{X}_{k}=\widehat{X}_{k, k-1}+K_{k} e_{k} \\
P_{k}=\left(I-K_{k} H_{k}\right) P_{k, k-1},
\end{gathered}
$$

where $e_{k}=Z_{k}-H_{k} \widehat{X}_{k, k-1}-r_{k}$.

The estimator of noise statistics of $q, Q, r$, and $R$ based on a maximum posteriori can be written as

$$
\begin{aligned}
\widehat{q}_{k}= & \left(1-\tau_{k}\right) \widehat{q}_{k-1}+\tau_{k}\left(B_{k}^{T} B_{k}\right)^{-1} B_{k}^{T}\left(\widehat{x}_{k-1}-\Phi_{k-1, k-2} \widehat{x}_{k-2}\right) \\
\widehat{Q}_{k}= & \left(1-\tau_{k}\right) \widehat{Q}_{k-1}+\left(B_{k}^{T} B_{k}\right)^{-1} B_{k}^{T} \tau_{k} \\
& \times\left(K_{k} e_{k} e_{k}^{T} K_{k}^{T}+P_{k}-\Phi_{k-1} P_{k-1} \Phi_{k-1}^{T}\right)\left[\left(B_{k}^{T} B_{k}\right)^{-1} B_{k}^{T}\right]^{T} \\
\widehat{r}_{k}= & \left(1-\tau_{k}\right) \widehat{r}_{k-1}+\tau_{k}\left(Z_{k}-H_{k} \widehat{x}_{k, k-1}\right) \\
\widehat{R}_{k}= & \left(1-\tau_{k}\right) \widehat{R}_{k-1}+\tau_{k}\left(e_{k} e_{k}^{T}-H_{k} P_{k, k-1} H_{k}^{T}\right),
\end{aligned}
$$

where $\tau_{k}=1 / k$.
2.2.2. The Proposed Method. In SHAKF, KF and adaptive noise estimator are carried out directly in time domain; the internal connecting and mutual coupling exist between the estimation of the state and the noise parameter. To this respect, it is easy to cause the instability and filtering accuracy reduction.

Based on the analysis of the frequency domain, system status is the integral of motivated white noise $w$, and the system noise is mainly characterized by noise of low frequency, such as ARW, when it is transferred to the measurement output. Meanwhile, the white noise $v$ of measurement is mainly characterized by broadband noise when it affects the measurement output directly. Therefore, the factor of measurement noise can be isolated based on the division of frequency band of measurement output. The Allan variance estimator is a band-pass filter, and quantization noise will be significantly reduced after increasing the rate of data sampling. To this respect, white noise variance can be approximately regarded as the Allan variance of broadband white noise in the present moment $\tau_{M}$

$$
\widehat{R}_{k} \approx \widehat{\sigma}_{k}^{2}\left(\tau_{M}\right) .
$$

It provides a feasible way to estimate variance of white noise of measurement. When the sampling interval is $\tau$, the Allan variance of FOG output in the moment is given by

$$
\widehat{\sigma}_{k}^{2}(\tau)=\frac{1}{2(N-1)} \sum_{i=2}^{k}\left[\bar{\Omega}_{i}(\tau)-\bar{\Omega}_{i-1}(\tau)\right]^{2},
$$

where $\bar{\Omega}_{i}(\tau), i=2,3, \ldots, k$ are samples of the average angular rate.

In order to simplify the analysis, it is considered that the components of measurement noise vector are irrelevant. In the step $k$ of $\mathrm{KF}$, it only needs to compute the Allan variance in the present moment of which the sample interval is the shortest sampling time $\tau_{0}$

$$
\begin{aligned}
\widehat{\sigma}_{k}^{2}\left(\tau_{0}\right)= & \frac{1}{2(k-1)} \sum_{i=2}^{k}\left(Z_{i}-Z_{i-1}\right)^{2} \\
= & \frac{1}{2(k-1)}\left[\sum_{i=2}^{k-1}\left(Z_{i}-Z_{i-1}\right)^{2}+\left(Z_{k}-Z_{k-1}\right)^{2}\right] \\
= & \left(1-\frac{1}{k-1}\right)\left[\frac{1}{2(k-2)} \sum_{i=2}^{k-1}\left(Z_{i}-Z_{i-1}\right)^{2}\right] \\
& +\frac{1}{2(k-1)}\left(Z_{k}-Z_{k-1}\right)^{2} .
\end{aligned}
$$

Therefore, the estimator of measurement noise can be rewritten into a recursive form as follows:

$$
\widehat{R}_{k}=\left(1-\frac{1}{k-1}\right) \widehat{R}_{k-1}+\frac{1}{2(k-1)}\left(Z_{k}-Z_{k-1}\right)^{2},
$$

where $k=2,3, \ldots$. The initial value of $\widehat{R}_{1}$ can take any value.

Considering the facts that the statistics of process noise and measurement noise are time-variant, an exponential 


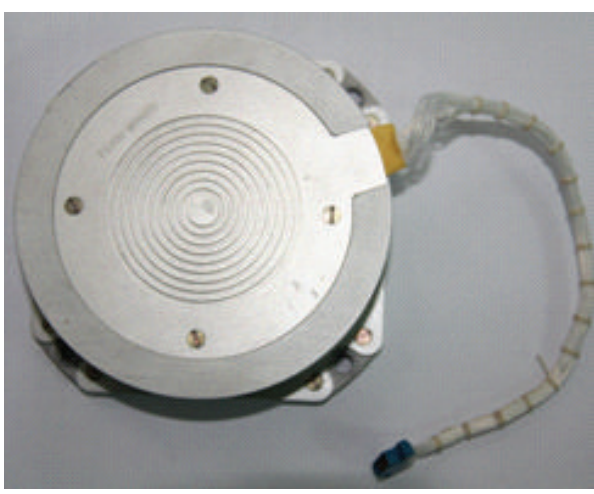

(a)

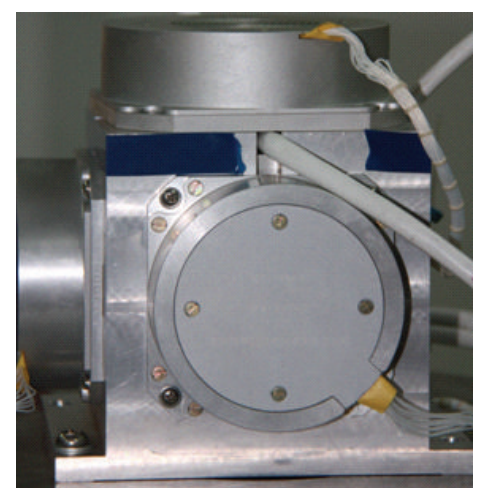

(b)

FIGURE 3: Procurement of FOG F120H and the processed IMU.

TABLE 2: Comparison of ARW estimation (digital simulation test, unit ${ }^{\circ} / \mathrm{h}^{1.5}$ ).

\begin{tabular}{|c|c|c|c|c|c|c|}
\hline \multirow{2}{*}{ Number } & \multirow{2}{*}{ Theoretical value } & \multirow{2}{*}{ Allan method } & \multicolumn{2}{|c|}{ SHAKF method } & \multicolumn{2}{|c|}{ The proposed method } \\
\hline & & & Mean & Variance & Mean & Variance \\
\hline 1 & 9.2000 & 9.1793 & 9.1268 & 0.0501 & 9.1561 & 0.0280 \\
\hline 2 & 1.5000 & 1.4980 & 1.4945 & 0.0041 & 1.4974 & 0.0011 \\
\hline 3 & 0.3800 & 0.3919 & 0.3783 & 0.0043 & 0.3795 & $2.5283 e-004$ \\
\hline 4 & 0.0810 & 0.0802 & 0.0841 & 0.0053 & 0.0942 & $3.3350 e-004$ \\
\hline 5 & 0.0320 & 0.0363 & 0.0801 & 0.0016 & 0.0349 & $5.0534 e-004$ \\
\hline
\end{tabular}

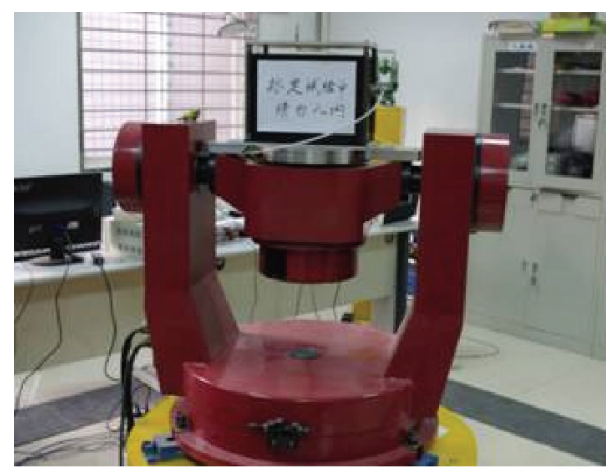

FIGURE 4: Laboratory biaxial rate turntable with high precision.

factor $\beta_{k}$ is brought in to strength the weights of recent information

$$
\beta_{k, i}=\frac{1-b}{1-b^{k}} b^{k-i}, \quad i=1,2, \ldots, k,
$$

where $\beta_{k, i}=\beta_{k, i-1} / b$ and $\sum_{i=1}^{k} \beta_{k, i}=1$ and the forgetting factor $b(0<b<1)$ is usually selected from $0.95 \sim 0.99$.

Then, the new estimator of measurement noise is obtained as follows by using $\beta_{k-1}$ instead of $1 /(k-1)$ in (16):

$$
\widehat{R}_{k}=\left(1-\beta_{k-1}\right) \widehat{R}_{k-1}+\frac{1}{2} \beta_{k-1}\left(Z_{k}-Z_{k-1}\right)^{2} .
$$

The new adaptive filter is presented by embedding the measurement noise estimator into the SHAKF framework. The processes of measurement noise variance estimation and
KF are independent of each other in the algorithm. Therefore, it can effectively reduce the problem of filtering divergence and reduction of precision.

\section{Experiments and Result}

In this section, the SHAKF method and the proposed method are tested for estimating ARW coefficient. The Allan variance method is also carried out to provide a basis for comparison. Firstly, these methods are tested using the digital simulation data. Then, they are tested on FOG experimental data.

3.1. Test by the Digital Simulation Data. The simulation data is used for the algorithm validating test in this section. In the simulation data, the ARW coefficient of FOG is set to $0.0810^{\circ} / \mathrm{h}^{1.5}, 0.3800^{\circ} / \mathrm{h}^{1.5}, 1.5000^{\circ} / \mathrm{h}^{1.5}$, and $9.2000^{\circ} / \mathrm{h}^{1.5}$, respectively.

For comparison, the recorded data is also analyzed using Allan variance method. From the Allan variance graph, the ARW coefficient is evaluated using least square fit method. The results of classical Allan variance, SHAKF, and the proposed online method are given in Table 2. The results of simulation test curve are shown in Figure 1, respectively.

The computational cost test is carried out, which is divided into two groups when the ARW coefficient of FOG is set to $0.3800^{\circ} / \mathrm{h}^{1.5}$ and $9.2000^{\circ} / \mathrm{h}^{1.5}$, respectively. The total calculation time of Allan method, SHAKF method, and the proposed method is intuitively shown in Figure 2 (calculation using the CPU of Intel Core2-P8400, 2.27 GHz). 


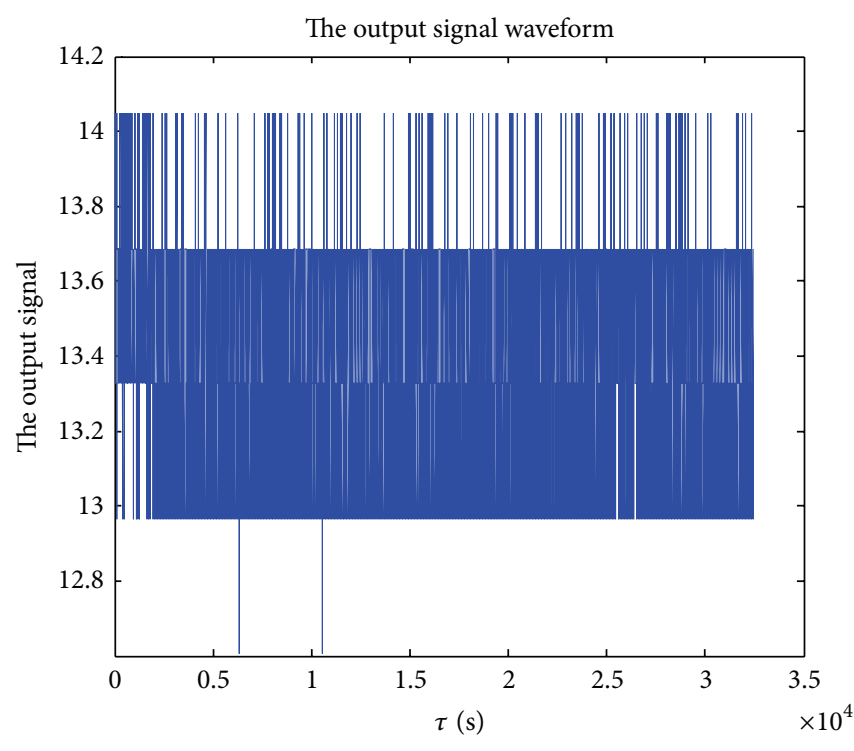

(a)

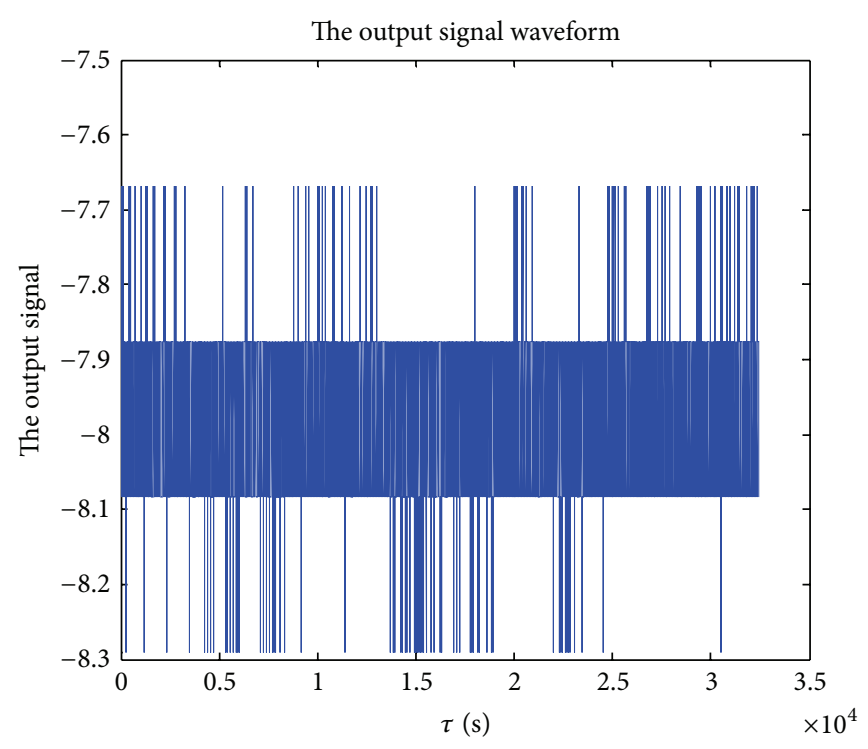

(b)

FIgURE 5: The output signal waveform of the $X, Z$ FOGs.

TABLE 3: Comparison of ARW estimation (experimental data test, unit $\left.^{\circ} / \mathrm{h}^{1.5}\right)$

\begin{tabular}{lccccc}
\hline \multirow{2}{*}{ FOG } & \multirow{2}{*}{ Allan method } & \multicolumn{2}{c}{ SHAKF method } & \multicolumn{2}{c}{ The proposed method } \\
& & Mean & Variance & Mean & Variance \\
\hline$X$-axis & 0.0651 & 0.0777 & 0.0119 & 0.0628 & 0.0010 \\
$Y$-axis & 0.0652 & 0.0772 & 0.0119 & 0.0628 & $6.1701 e-004$ \\
$Z$-axis & 0.0336 & 0.0402 & 0.0122 & 0.0330 & $7.8300 e-004$ \\
\hline
\end{tabular}

Figure 1 and Table 2 show that Allan variance method, SHAKF method, and the proposed online method can accurately estimate the ARW of FOG in different setting conditions. It can be seen that the accuracy of the proposed online method and the classical Allan variance method is basically identical. Especially it can be seen, from Figures $1(\mathrm{~d})$ to $1(\mathrm{e})$, that when the range of ARW is less than twice magnitude, the accuracy of the proposed method is slightly better than the SHAKF and the stability of filtering is stronger. Figure 2 shows that the proposed method outperforms Allan variance method in terms of the computational cost in simulation test. Note that the proposed method spends a little more time in estimation compared with SHAKF. The reason is that the delicate designed estimator of measurement noise is embedded into the framework of SHAKF and it will cost a little extra time in the calculation of the estimator.

3.2. Test by FOG Experimental Data. Currently, the laboratory has a FOG $(\mathrm{F} 120 \mathrm{H})$ for $X$-axis and two other FOGs $(\mathrm{F} 98 \mathrm{H})$ for $Y$ and $Z$-axis, and the bias stabilities are $0.02^{\circ} / \mathrm{h}$ and $0.04^{\circ} / \mathrm{h}$, respectively. In the paper, the test is carried out by using the experimental data at $100 \mathrm{~Hz}$ of $\mathrm{F} 120 \mathrm{H}$ and F98H as shown in Figure 3. FOGs are installed in biaxial rate turntable as shown in Figure 4. The output-signal waveforms of the $X, Z$ FOGs are shown in Figure 5, respectively.
The results of classical Allan variance, SHAKF method, and the proposed online method are shown in Table 3. The results of simulation test curve are shown in Figure 6, respectively. The total calculation time of these methods is intuitively shown in Figure 7 (calculation using the CPU of Intel Core2-P8400, 2.27 GHz).

With reference to the estimated value by Allan variance method, Table 3 and Figure 6 show that the proposed method can estimate the ARW of $X, Y, Z$ FOGs online in practical application. The relative errors of the estimation of ARW of $X, Y, Z$ FOGs by the proposed online method relative to the Allan variance method are $3.51 \%, 3.70 \%$, and $1.79 \%$, respectively. By comparison, the relative errors of the SHAKF method are $19.35 \%, 18.41 \%$, and $19.64 \%$. Therefore, to some extent, the SHAKF method can estimate the ARW; however, the estimated accuracy and the rate of convergence are not ideal in practice. Figure 7 shows intuitively that the proposed method outperforms the Allan variance method in terms of the computational cost in field test.

\section{Conclusion}

As a standard method for noise analysis of FOG, Allan variance method is offline and requires storing a large number of hours of FOG output data. A new online method based on the SHAKF is proposed in the paper for estimating the ARW coefficient of FOG. The main innovation is based on embedding the delicate designed estimator of measurement noise into the framework of SHAKF that performs better in general online applications. The proposed method can analyze data as it arrives from onboard FOG and estimate ARW coefficient online. Therefore, it does not require large amounts of data storage or manual analysis for an Allan variance graph. Experimental results including the digital simulation test and experimental data test of FOG show 

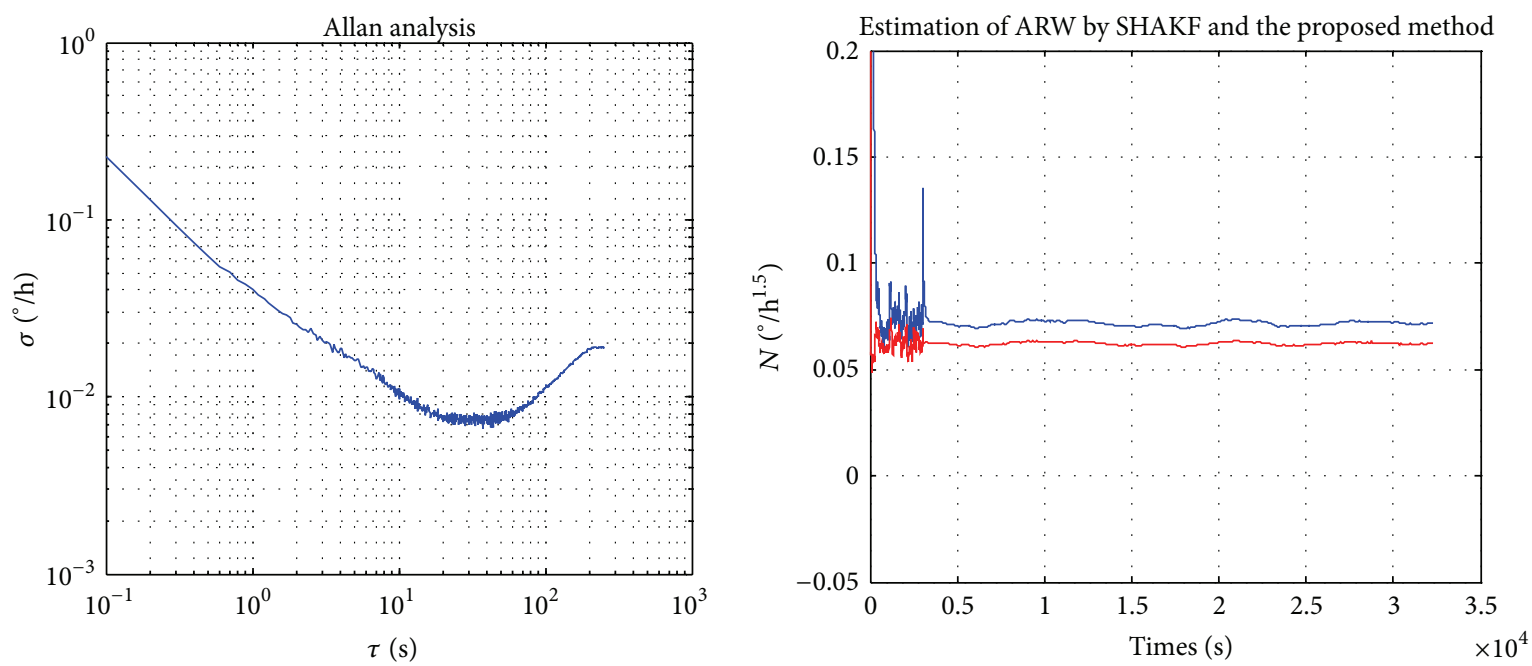

(a) $X$ Axis
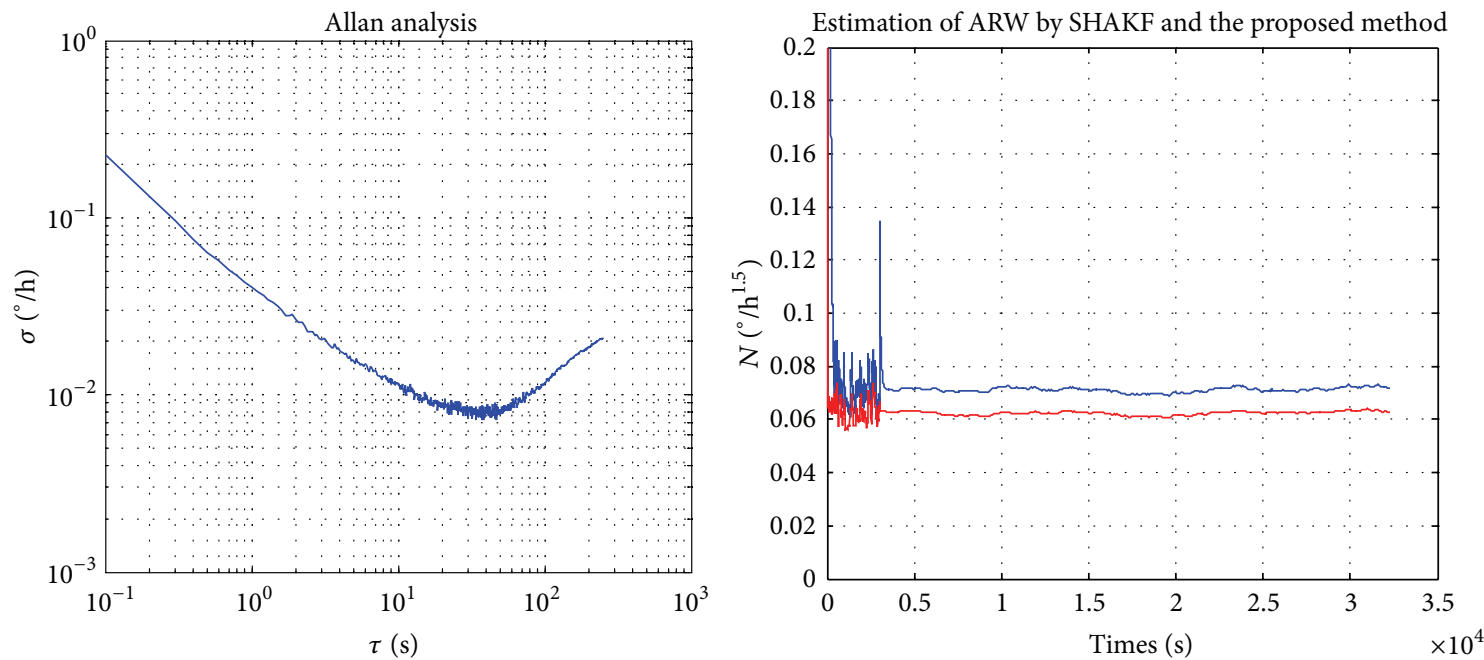

(b) $Y$ Axis
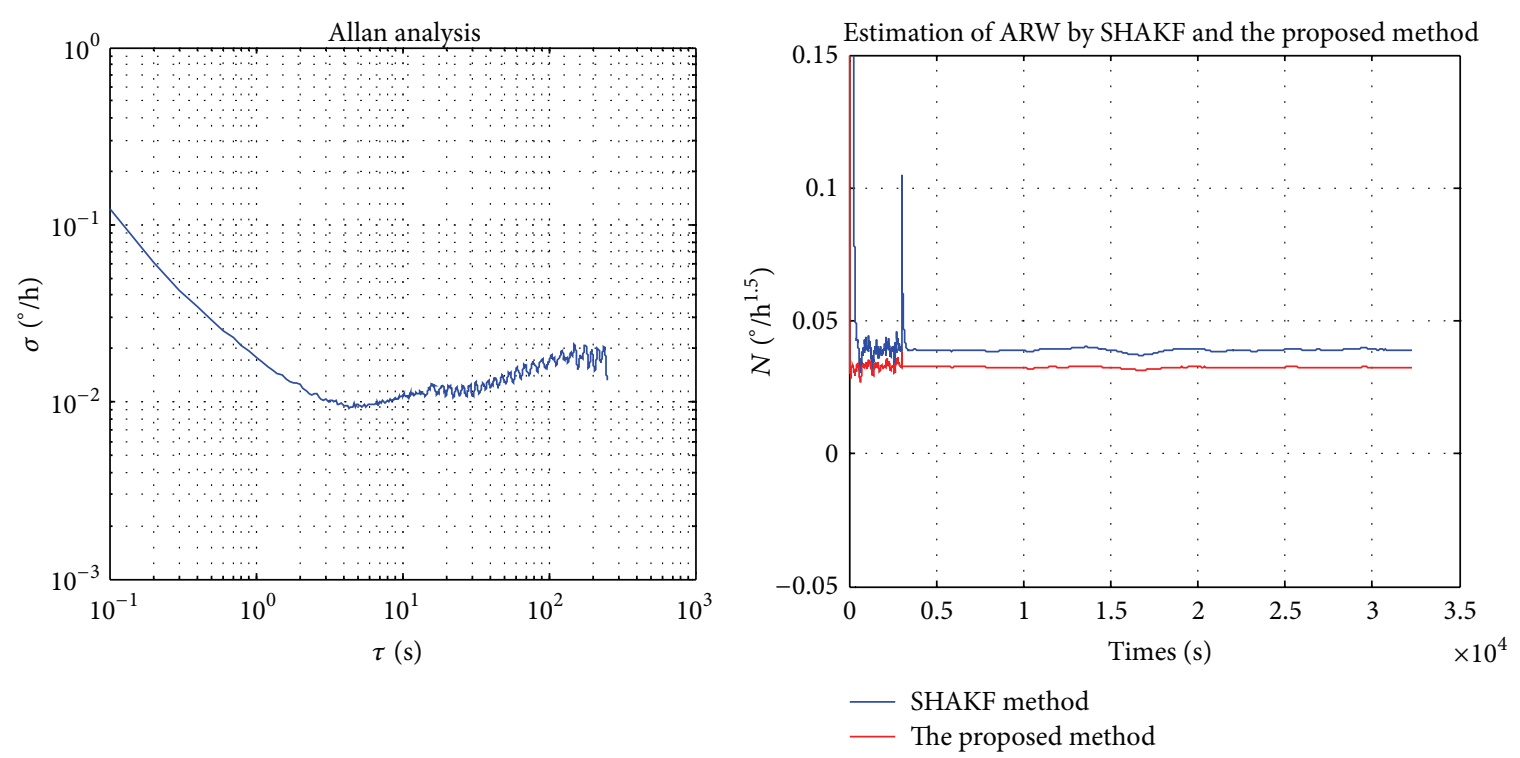

(c) Z Axis

FIGURE 6: Comparison of ARW estimation of different FOGs (experimental data test). 


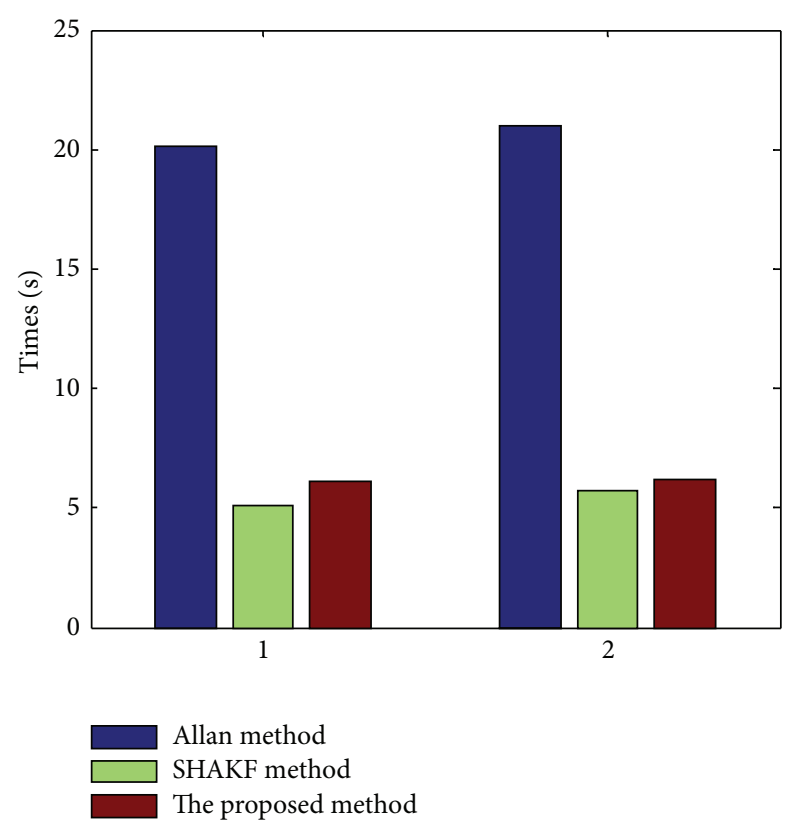

Figure 7: Comparison of total calculation time (1: $X$ FOG, $2: Z$ FOG).

that the proposed method based on SHAKF framework is basically identical in terms of accuracy compared with Allan variance method and outperforms Allan variance method in terms of computational cost. Meanwhile, it can be seen from the test data that the proposed online method in the paper making use of only a little more time can achieve more accurate performance than the SHAKF method, which further demonstrates the effectiveness of the proposed method.

\section{Conflict of Interests}

The authors declare that there is no conflict of interests regarding the publication of this paper.

\section{Acknowledgment}

This work is supported by the National Natural Science Foundation of China (no. 61304241, no. 61374206, and no. 61104184).

\section{References}

[1] Q. M. Lam, N. Stamatakos, C. Woodruff, and S. Ashton, "Gyro modeling and estimation of its random noise sources," in AIAA Guidance, Navigation, and Control Conference and Exhibit, pp. 1-2, AIAA Inc, Austin, Tex, USA, August 2003.

[2] "IEEE Standard Specification Format Guide and Test Procedure for Single-Axis Interferometric Fiber Optic Gyros," IEEE Standard 952-1997(R2008), pp. 166-181, 2008.

[3] T. J. Witt, "Using the allan variance and power spectral density to characterize DC nanovoltmeters," IEEE Transactions on Instrumentation and Measurement, vol. 50, no. 2, pp. 445-448, 2001.
[4] J. J. Ford and M. E. Evans, "Online estimation of allan variance parameters," Journal of Guidance, Control, and Dynamics, vol. 23, no. 6, pp. 980-987, 2000.

[5] N. El-Sheimy, H. Hou, and X. Niu, "Analysis and modeling of inertial sensors using allan variance," IEEE Transactions on Instrumentation and Measurement, vol. 57, no. 1, pp. 140-149, 2008.

[6] B. E. Grantham and M. A. Bailey, "A least-squares normalized error regression algorithm with application to the allan variance noise analysis method," in Proceedings of the IEEE/ION Position, Location, and Navigation Symposium, pp. 750-756, April 2006.

[7] O. Filla, T. Willard, D. Chu, and J. Deutschmann, "Inflight estimation of gyro noise," in Flight Mechanics/Estimation Theory Symposium, pp. 41-58, 58, Greenbelt, Md, USA, 1990.

[8] Q. M. Lam, T. Wilson Jr., R. Contillo, and D. Buck, "Enhancing MEMS sensors accuracy via random noise characterization and calibration," in Sensors, and Command, Control, Communications, and Intelligence (C31) Technologies for Homeland Security and Homeland Defense III, vol. 5403 of Proceedings of SPIE, pp. 427-438, Orlando, Fla, USA, April 2004.

[9] N. Song, R. Yuan, and J. Jin, "Autonomous estimation of Allan variance coefficients of onboard fiber optic gyro," Journal of Instrumentation, vol. 6, no. 9, Article ID P09005, pp. 2-10, 2011.

[10] V. Saini, S. C. Rana, and M. M. Kuber, "Online estimation of state space error model for MEMS IMU," Journal of Modelling and Simulation of Systems, vol. 1, no. 4, pp. 219-225, 2010.

[11] B. Hu, L. Chang, A. Li, and F. Qin, "Comment on "highly efficient sigma point filter for spacecraft attitude and rate estimation'"' Mathematical Problems in Engineering, vol. 2012, Article ID 170391, 5 pages, 2012.

[12] S. Y. Chen, "Kalman filter for robot vision: a survey," IEEE Transactions on Industrial Electronics, vol. 59, no. 11, pp. 44094420, 2012.

[13] L. Chang, B. Hu, A. Li, and F. Qin, "Transformed unscented Kalman filter," IEEE Transactions on Automatic Control, vol. 58, no. 1, pp. 252-257, 2013.

[14] D. Y. Kim and M. Jeon, "Robust distributed Kalman filter for wireless sensor networks with uncertain communication channels," Mathematical Problems in Engineering, vol. 2012, Article ID 238597, 12 pages, 2012.

[15] K. A. Myers and B. D. Tapley, "Adaptive sequential estimation with unknown noise statistics," IEEE Transactions on Automatic Control, vol. 21, no. 4, pp. 520-523, 1976.

[16] A. P. Sage and G. W. Husa, "Adaptive filtering with unknown prior statistics," in Proceedings of the Joint Automatic Control Conference, pp. 760-769, 1969.

[17] A. H. Mohamed and K. P. Schwarz, "Adaptive Kalman filtering for INS/GPS," Journal of Geodesy, vol. 73, no. 4, pp. 193-203, 1999.

[18] R. K. Mehra, “Approaches to adaptive filtering," IEEE Transactions on Automatic Control, vol. 17, no. 5, pp. 693-698, 1972.

[19] W. Gao and J. C. Li, "Adaptive Kalman filtering for the integrated SINS/DVL system," Journal of Computational Information Systems, vol. 16, no. 9, pp. 6443-6450, 2013.

[20] L. B. Chang, B. Q. Hu, A. Li, and F. J. Qin, "Strapdown inertial navigation system alignment based on marginalized unscented Kalman filter," IET Science, Measurement and Technology, vol. 7, no. 2, pp. 128-138, 2013.

[21] M. Karasalo and X. Hu, "An optimization approach to adaptive Kalman filtering," Automatica, vol. 47, no. 8, pp. 1785-1793, 2011. 
[22] H. Qian, Q. Xia, B. Jiang, and C. Wang, "On modeling of random drift of MEMS gyroscope and design of kalman filter," in Proceedings of the IEEE International Conference on Mechatronics and Automation (ICMA '09), pp. 4355-4360, August 2009. 


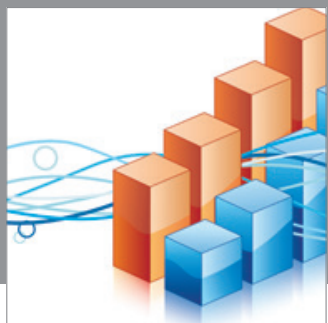

Advances in

Operations Research

mansans

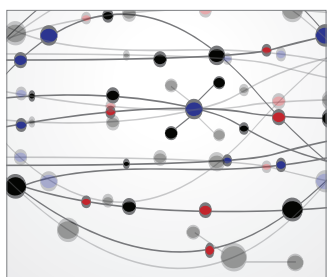

The Scientific World Journal
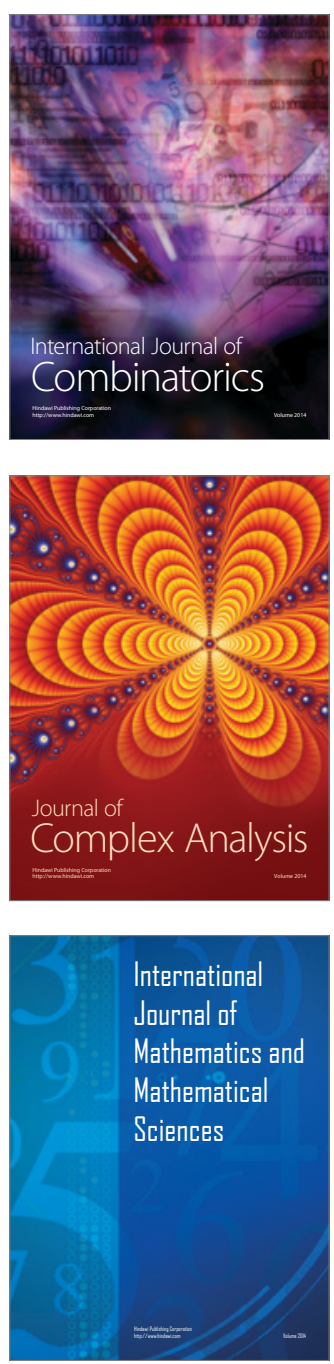
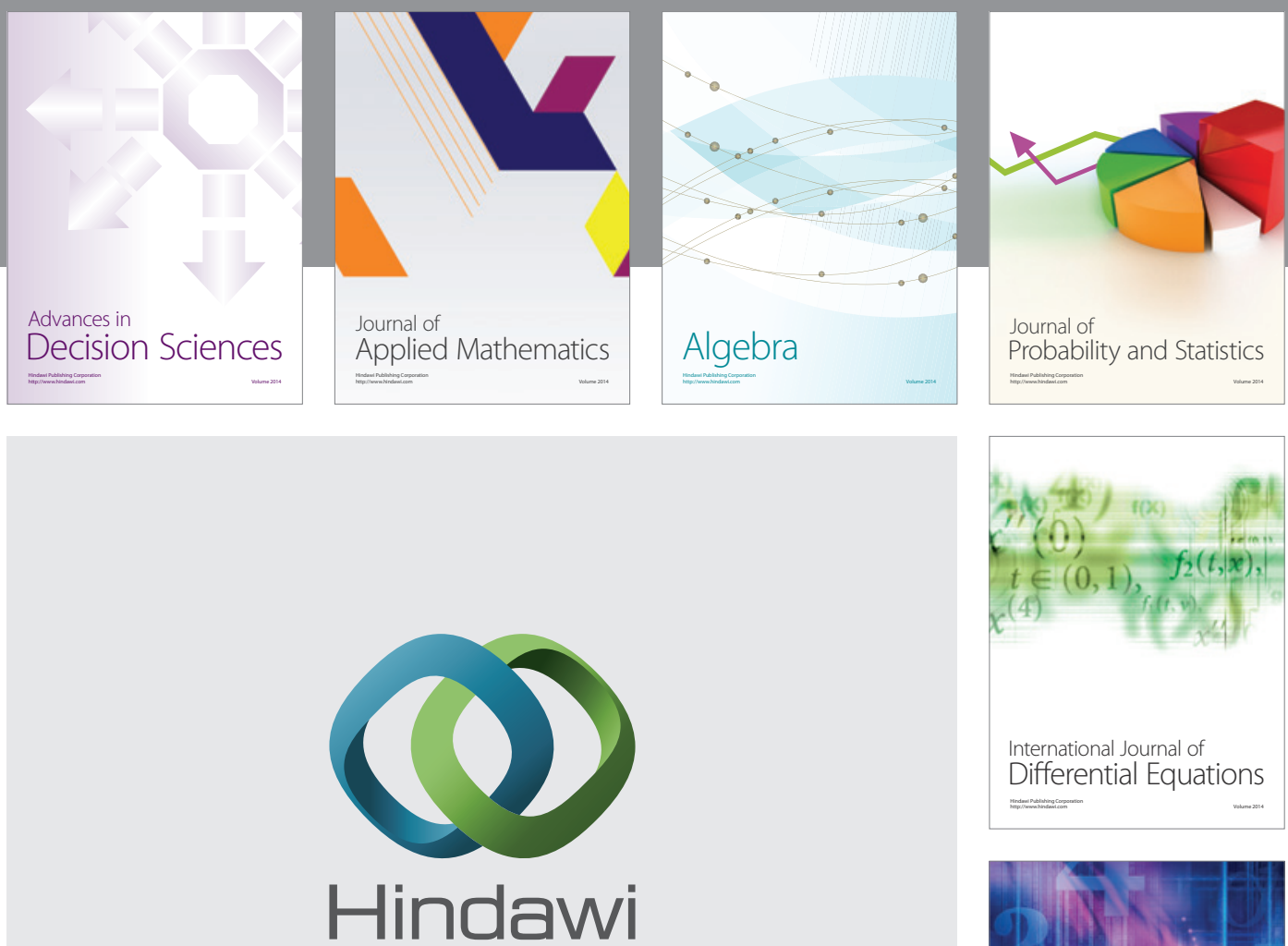

Submit your manuscripts at http://www.hindawi.com
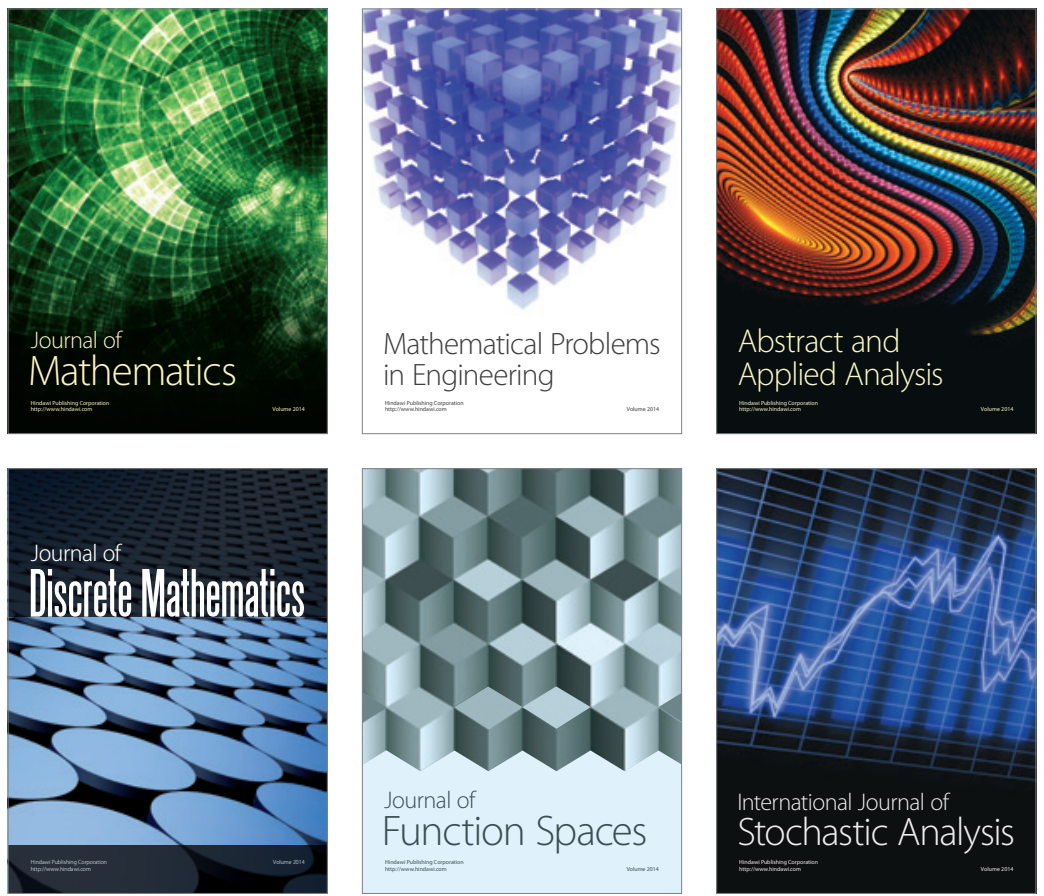

Journal of

Function Spaces

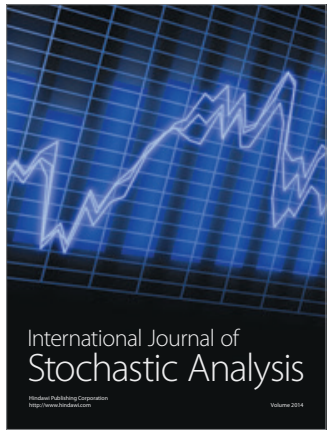

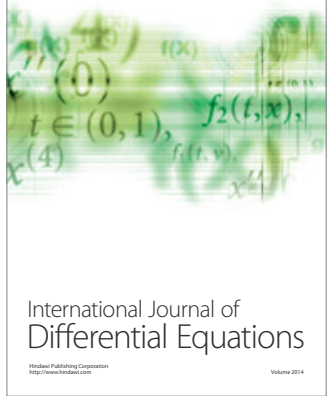
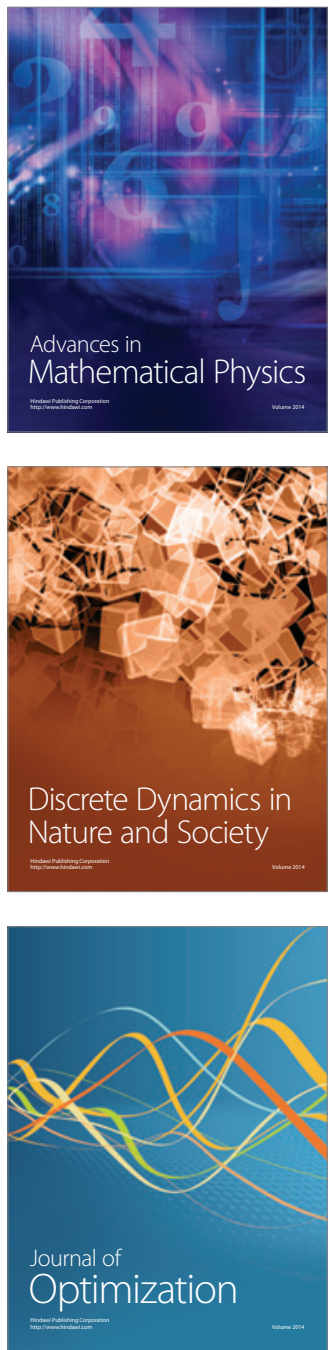